\title{
Home-Office: Aspectos Exploratórios do Trabalho a partir de Casa
}

\author{
Julia Carolina Rafalski ${ }^{1}$ \\ Programa de Pós-Graduação em Psicologia da Universidade Federal do Espírito Santo, \\ Vitória, ES, Brasil \\ Alexsandro Luiz De Andrade \\ Departamento de Psicologia da Universidade Federal do Espírito Santo, Vitória, ES, Brasil \\ Programa de Pós-Graduação em Psicologia da Universidade Federal do Espírito Santo, \\ Vitória, ES, Brasil
}

\begin{abstract}
Resumo
Novas configurações no mundo global imprimem diferentes modificações nos processos de trabalho, rotinas e gestão da informação. Entre as novas formas de trabalho surgidas na década de 70 do último século está o home-office, trabalho realizado a partir da própria residência, que tem obtido maior proeminência nos últimos anos. A presente pesquisa visa conhecer aspectos de trabalho e variáveis psicossociais que circundam 74 profissionais brasileiros ( $M=38,2$ anos, $D P=8,8$ anos) que relataram trabalhar ou terem trabalhado em home-office. Participantes responderam um questionário com perguntas abertas, cujas respostas foram analisadas por meio de análise de conteúdo e de frequência. Como resultados verificou-se que 62 participantes atuavam em home-office no momento da pesquisa, sendo a principal vantagem citada deste sistema de trabalho a flexibilidade de horários e a desvantagem mais recorrente a falta de socialização. Foram mencionados problemas em termos profissionais e pessoais, como implicações para a saúde e para a vida familiar dos trabalhadores, além de terem sido expostos contrapontos positivos para os mesmos âmbitos. São necessários estudos adicionais para ampliar conhecimentos neste campo, uma vez que se trata de pesquisa inicial no Brasil.
\end{abstract}

Palavras-chave: Trabalho, home-office, autonomia e teletrabalho.

\section{Home-Office: Exploratory Aspects of Work from Home}

\begin{abstract}
New global configurations produce different modifications on work, routine and information management procedures. The home-office, work made from home, is among new work strategies that arose in 1970's, which achieved greater prominence in recent years. This paper aims to understand aspects of work and psychosocial variables surrounding 74 professionals $(M=38.2$ e $S D=8.8)$ that reported to work or have worked in home-office. Participants answered an online questionnaire with open and closed questions, which responses were analyzed with content analysis and frequency. Results show that 62 participants worked from home-office at the moment of the research, being the main advantage of this model of work the flexibility of hours and the most recurrent disadvantage was the lack of socialization. Participants cited problems in professional and personal terms, such as implications for health and family life and

Endereço para correspondência: Av. Fernando Ferrari, 514 (CEMUNI IV) - Goiabeiras/Vitória, ES -Brasil | CEP 29075-910. E-mail: jcrafalski@gmail.com e alexsandro.deandrade@yahoo.com Agência de financiamento: Conselho Nacional de Desenvolvimento Científico e Tecnológico (CNPq).
\end{abstract}


also exposed positive counterpoints in the same areas. As an exploratory research, additional studies are required to expand the knowledge in this field in Brazil.

Keywords: Work, home-office, autonomy and telework.

\section{Teletrabajo: Aspectos Exploratorios de Trabajar desde Casa}

\section{Resumen}

Nuevos valores en el mundo global se imprimen diferentes cambios en los procesos de trabajo, rutinas y gestión de la información. Entre las nuevas formas de trabajo que surgieron en la década de los 70 la oficina en casa, trabajar desde la propia residencia, que ha conseguido más importancia en los últimos años. Esta investigación tiene como objetivo estudiar los aspectos del trabajo y las variables psicosociales que rodean 74 profesionales brasileños $(M=38.2$ años, $S D=8.8$ años $)$ que declararon trabajar o han trabajado en la casa-oficina. Los participantes respondieron un cuestionario con preguntas abiertas, cuyas respuestas se analizaron mediante análisis de contenido y la frecuencia. Como resultado, se encontró que 62 participantes trabajaron en la oficina en casa en el momento de la encuesta, la principal ventaja en este sistema de trabajo fue horarios flexibles y la principal desventaja citaron fue la falta de socialización. Los problemas en términos profesionales y personales, se mencionaron las consecuencias para la salud y la vida familiar de los trabajadores, así como contrapuntos positivos han estado expuestos a los mismos niveles. Debido a que esta investigación inicial en Brasil, se necesitan más estudios para ampliar los conocimientos en este campo.

Palabras clave: Trabajo, home-office, autonomía y teletrabajo.

Percebe-se, na atualidade, muito em parte pelo advento da globalização, novas configurações sociais, econômicas e tecnológicas, as quais fazem emergir profundas modificações no mundo trabalho. São exemplos dessas modificações a flexibilização da produção, a terceirização da mão de obra, a produção just-in-time, modelos de carreiras com características mais individuais e a maior valorização do capital humano e psicológico no trabalho. A abertura do mercado em países em desenvolvimento para empresas multinacionais e a forma de trabalho em home-office, possibilitada pela internacionalização e descentralização das empresas, caracterizam um cenário com diferentes formas de trabalhar e se apresentam como uma realidade do fenômeno trabalho.

O trabalho é um produto de diferentes elementos históricos, políticos e sociais e, como objeto de estudo, se encontra em um cenário de diferentes áreas de saber, sujeito a interferências de mudanças em todos seus âmbitos. Os trabalhadores e as organizações, por consequência, precisam se adaptar aos novos modos de desen- volvimento de tarefas, conforme esses surgem e se estabelecem. Desse modo, tornam a flexibilidade e a capacidade de suportar as mudanças frequentes nos modos de gestão, em habilidades necessárias à sobrevivência profissional (Baruch, 2001; Rosa, 2010). Como exemplo, ainda que após os anos 2000 o trabalho em casa esteja crescendo em popularidade, tem-se conhecimento de performances profissionais realizadas em plataformas móveis ou escritórios-satélites desde a década de 1970, sendo que tais iniciativas já apresentavam o objetivo de diminuir custos e aumentar o conforto do trabalhador (Pratt, 1984).

Mesmo com o desenvolvimento crescente de pesquisas que investigam a atuação do trabalhador à distância de seu ambiente profissional, não existe um termo geral utilizado com concordância entre os pesquisadores, ocorrendo a utilização dos diferentes termos como sinônimos, muitas vezes (Baruch, 2001). Pratt (1984) diferencia os termos 'telecomutação' (no original, 'telecommuting'), 'teletrabalho' e 'local-flexível' (no original, 'flexiplace') quanto ao local de realização e ao uso de ferramentas de computa- 
ção para o cumprimento das atividades laborais. Para o autor, embora os termos teletrabalho e local-flexível explicitem que não há necessidade de se estar no escritório formal, o segundo, adicionalmente, não inclui a necessidade do uso de ferramentas computacionais, enquanto o termo telecomutação exporia o uso de tecnologia para substituir a ida diária ao escritório.

Di Martino e Wirth (1990) apontam que o teletrabalho pode ser definido como o conjunto de atividades profissionais realizadas fora do escritório/da empresa, sem contato pessoal com colegas de trabalho, mas com a possibilidade de se comunicar com estes por meio de tecnologia de qualquer tipo (exemplo: telefone móvel ou e-mails). Pode, ainda, ser realizado online ou off-line, referindo-se ao uso direto dos computadores, mostrando ser uma definição mais ampla. Pode assumir a forma do trabalho realizado de casa (work from home-telework-home-office), por uma época ou de forma determinada e permanente, sendo um erro considerá-lo somente descritivo do trabalho em situação de emprego formal (Huws, Korte, \& Robinson, 1990). Em definição ainda mais ampla, o teletrabalho pode ser caracterizado quando os trabalhadores realizam todas ou grande parte de suas atividades em um ambiente fisicamente separado do local de seu trabalho, usando meios tecnológicos para sua realização (Baruch, 2001; Konradt, Schmook, \& Mälecke, 2000).

A modalidade de trabalho home-office se caracteriza pela inserção em um espaço, sem tamanho ou propriedades definidas, destinado somente a atividades profissionais e localizado na residência do trabalhador, sendo facultativo o uso de tecnologias informacionais para a realização das atividades (Ellison, 1999; Lim \& Teo, 2000). Para Mendonça (2010), a nomenclatura do home-office é exclusividade do uso do local residencial, mesmo que partilhado por outros moradores. Explicita, também, que as atividades possuem horários estabelecidos de forma mais ou menos flexível e são de cunho profissional, sejam elas de empresários/autônomos ou ligadas a uma organização privada, como, por exemplo, de prestação de serviços. Para fins de definição deste estudo, trabalhar em home-office se carac- teriza por desempenhar as atividades profissionais no mesmo ambiente em que se reside.

O trabalho em home-office e o teletrabalho, em geral, têm se disseminado como meios de trabalho eficientes e viáveis. São considerados modelos adaptados aos avanços tecnológicos e equivalentes quanto aos ganhos e às perdas de empregados e empregadores; um modo de trabalho a ser amplamente realizado 'no futuro' (Rasmussen \& Corbett, 2008; Ward \& Shabba, 2001). Segundo Pyöriä (2011), entretanto, essa difusão do novo modelo de trabalho tem acontecido em um processo mais lento do que o esperado, devido, principalmente, à falta de cultura empresarial para gerenciamentos à distância.

$\mathrm{Na}$ área do Direito do Trabalho, Duarte (2006) expõe que trabalhar em domicílio, desde que subordinado a um empregador ou respaldado por uma empresa, insere-se, para todos os efeitos, como um trabalho em regime de Consolidação das Leis do Trabalho (CLT). O trabalhador de home-office responde aos superiores em sua empresa como se estivesse inserido fisicamente no ambiente laboral, sem distinção legal do trabalho considerado formal, seja em relação ao salário ou aos benefícios, sendo concedidos, de forma igualitária, as férias e o $13^{\circ}$ salário, bem como demais gratificações. Rasmussen e Corbett (2008) pontuam que a falta de direitos concedidos ao trabalho em home-office e a informalidade deste modelo podem contribuir para uma reputação negativa, podendo gerar desqualificação profissional. Jaakson e Kallaste (2010), neste sentido, discutem a responsabilidade conferida aos trabalhadores e aos empregadores, sinalizando que parte das condições em que se realizam este trabalho são mutuamente acordadas, ainda que não válidas em nível legal.

Uma vez que o home-office é entendido como uma maneira de redução de custos organizacionais (Pratt, 1984), é importante que a economia feita pelas empresas possa ser utilizada como recurso para facilitar a adaptação do trabalhador ao home-office, proporcionando menor influência no ambiente e na família e, ao mesmo tempo, promovendo condições adequadas ao exercício da profissão ( $\mathrm{Ng}$, 2010). Rasmussen e Corbett (2008) apontam que podem ocorrer consequên- 
cias significativas devido à má adaptação ao novo ambiente de trabalho, como a falta de planejamento das atividades e a sensação de isolamento e o distanciamento da empresa, que contribui para diminuir a segurança profissional dos trabalhadores. Ward e Shabba (2001), por sua vez, dividiram os fatores que podem influenciar no comportamento organizacional em dois aspectos - psicológico e social -, apontando que problemas relativos ao distanciamento de outros profissionais são comuns neste modelo de trabalho.

Sardeshmukh, Sharma e Golden (2012), em pesquisa com 417 teletrabalhadores e home-officers, observaram que os principais efeitos positivos deste modelo de trabalho são a redução da pressão, a diminuição do conflito entre papéis de trabalho e a maior autonomia. Em contrapartida, foram identificados efeitos negativos como o aumento da ambiguidade profissional e a redução do suporte e do feedback. Foram obtidos, também, dados indicativos de que sentimentos de solidão, irritabilidade, preocupação, culpa e estresse estavam associados aos impactos negativos do trabalho em home-office (Golden, Veiga, \& Dino, 2008; Mann \& Holdsworth, 2003; Pratt, 1984; Ward \& Shabba, 2001).

Neste contexto, esta pesquisa possui por objetivo explorar as implicações sociais e psicológicas dos aspectos de trabalho que norteiam a atuação em home-office. Como destacam Bastos, Rodrigues, Moscon, Costa e Pinho (2013), novas formas de trabalho ainda são pouco estudadas. Entre estas tem-se o home-office, além do teletrabalho e as formas de trabalho com contratos temporários. Os poucos estudos brasileiros disponíveis sobre esta temática se alocam em dissertações, teses e trabalhos publicados em anais de eventos científicos. Tendo em vista que há carência de estudos empíricos com a população brasileira, esta pesquisa objetiva ampliar os conhecimentos acerca deste novo modo de configuração do trabalho, possibilitando uma base para a produção de futuros trabalhos, a obtenção de conhecimentos mais aprofundados, além da elaboração de estratégias de suporte à inserção e à adaptação destes trabalhadores ao ambiente laboral em casa.

\section{Método}

\section{Participantes}

Participaram deste estudo 74 pessoas que declararam terem atuado ou estarem atuando em home-office no momento da coleta, sendo 28 destes participantes do sexo feminino. A média da idade dos participantes foi de 38,2 anos ( $D P=$ 8,8 anos), com idades entre 23 e 67 anos. Em relação à distribuição geográfica, grande parte é originária da Região Sudeste do Brasil, com 53 participantes $(71,6 \%)$; não houve participantes oriundos da Região Norte do país.

Em relação ao seu atual status de trabalho, 62 participantes relataram que atuavam em home-office no momento da pesquisa. Desses, 32 participantes encontravam-se neste modelo de trabalho há menos de 2 anos, 16 participantes trabalham neste modelo entre 2 e 6 anos e 6 participantes relataram atuar há mais de 7 anos. Entre os participantes que relataram estar nesse modelo, 8 especificaram que sua atuação é esporádica, acontecendo primordialmente nos momentos entre viagens e nos finais de semana. Dentre os participantes que sinalizaram atuar em home-office, $52,7 \%$ destes (39 participantes) se encontram em seu primeiro trabalho nessa modalidade. Quanto às principais áreas de atuação da amostra têm-se: Mídias Sociais e Publicidade (24,4\%), Desenvolvimento e Análise de Sistemas (19\%), Comercial e Vendas $(17,5 \%)$, Administração (16,2\%), Consultorias em geral $(16,2 \%)$ e outros cargos $(6,7 \%)$.

\section{Instrumento e Procedimentos}

Optou-se por elaborar um questionário com questões abertas, disposto de forma online para acessar participantes de diferentes regiões do território nacional, possibilitando maior abrangência do estudo. Conforme Wachelke e de Andrade (2009), a coleta de dados online possibilita maior viabilidade no recrutamento de participantes, além de permitir que pesquisadores com acesso limitado a recursos financeiros possam produzir e contribuir cientificamente. De acordo com as normas éticas brasileiras para realização 
de pesquisa com seres humanos (Conselho Nacional de Saúde [CNS] 466/12), os participantes foram informados do caráter anônimo e voluntário da pesquisa, por meio do Termo de Consentimento Livre e Esclarecido.

O questionário elaborado continha 14 perguntas acerca da configuração do trabalho em home-office dos respondentes e sobre os aspectos sociais e psicológicos diretamente ligados ao trabalho, solicitando aos participantes que fornecessem respostas descritivas e explicativas. Os questionamentos foram elaborados com base na literatura acerca do tema, visando conhecer quem são os home-officers, por que fazem este trabalho, o que fazem e o que resulta de seu trabalho, além dos quatro principais círculos temáticos: a inserção e adaptação ao home-office, as implicações profissionais, os impactos na vida social e as questões psicológicas (Bailey \& Kurland, 2002; Pratt, 1984). Os participantes foram questionados, também, sobre mudanças significativas que ocorreram em suas vidas pessoais e profissionais após o início do trabalho nesse modelo, bem como as vantagens e desvantagens dessa atuação.

O recrutamento dos participantes foi realizado a partir de convites de divulgação via e-mail e em redes sociais, como Facebook e LinkedIn, fato que possibilitou a maior abrangência do estudo em território brasileiro. Para garantir a validade dos dados coletados, foram registrados os endereços de IP, data e hora de preenchimento de cada formulário, possibilitando a exclusão de dados duplicados ou enviados a partir de um mesmo registro, evitando, assim, fraudes e repetições.

\section{Procedimentos de Análise dos Dados}

Para a análise dos dados coletados foi realizada análise de conteúdo das questões abertas (Bardin, 1997; Strauss \& Corbin, 1998), além do cálculo de frequência de ocorrência dos temas. Cada questão do questionário foi analisada separadamente, a partir de categorias criadas por proximidade de temas, entre os respondentes e suas respostas em cada questionamento. Os elementos que emergiram em comum nas conside- rações dos participantes foram posteriormente agrupados e somados, em termos de frequência de ocorrência. Foram feitas análises categóricas, tomando como base aspectos sociodemográfi$\cos$, para estabelecimento de algumas caracterizações e diferenciações sobre o fenômeno.

\section{Resultados e Discussão}

São expostos a seguir os resultados do estudo realizado. Para melhor organização dos dados optou-se por uma sistematização em torno dos seguintes aspectos: Facilidades e vantagens do home-office e os aspectos de Transição e os desafios do trabalho a partir de casa. Respectivamente, são levantados os aspectos positivos do modo de gestão do trabalho a partir de casa e, então, explicitadas as características transitórias para o modelo home-office, além das principais dificuldades dos trabalhadores que se engajaram neste tipo de prestação serviço.

\section{Facilidades e Vantagens do Home-Office}

O primeiro ponto descritivo dos dados do estudo diz respeito ao motivo pelo qual os participantes optaram pelo trabalho em home-office. Os temas que emergiram das respostas sinalizam uma diferenciação entre os trabalhadores vinculados a empresas e os trabalhadores que optaram por abrir seus próprios negócios, atuando de maneira autônoma. O primeiro grupo é composto por 28 participantes-trabalhadores vinculados a empresas, que relataram terem sido convidados ou terem solicitado o ingresso na modalidade home-office. Para esses respondentes, a otimização do tempo, a obtenção de independência, a maturidade profissional, a diminuição de custos pessoais, o aumento de produtividade, e a redução do tempo no trânsito foram fatores relevantes para a aceitação da mudança de sistema de trabalho. Para Mello (1999), realizar as atividades de trabalho em domicílio ou local intermediário possibilita maior competitividade consigo mesmo e flexibilidade nas rotinas e horários, sendo estes pontos favoráveis à adoção desse regime por empresas de diferentes segmentos. Em pes- 
quisa com mais de 23 mil canadenses, por meio da Workplace Employment Survey, Tremblay, Paquet e Najem (2006) observaram que dois terços dos respondentes indicaram terem entrado no modelo home-office devido a questões de trabalho e não questões ligadas à família. Em contraponto, 60 empresas pesquisadas indicam que seus motivos abarcam, principalmente, redução de custos, reestruturação organizacional e aumento de produtividade (Konradt et al., 2000).

O segundo grupo, de trabalhadores que optaram por atuar em regime home-office devido à criação de sua empresa própria, prestação de consultorias ou trabalhos como freelancer, foi formado por 15 participantes. Estes consideram que a redução de custos, a flexibilização nos horários de trabalho e o aumento da produtividade são os principais fatores que propiciaram esta escolha. Duarte (2006) pontua que, em face das mudanças tecnológicas, a prestação de serviços se tornou mais cômoda e útil, tanto para empregados quanto para empregadores, que podem utilizar de suas capacidades intelectivas sem a necessidade de outro espaço físico, minimizando custos. De forma geral, os principais temas explicitados envolveram questões de produtividade, organização do tempo e independência. As respostas corroboram dados indicativos de que as motivações de trabalhadores para ingressarem em home-office giram em torno de 3 fatores: motivos econômicos e financeiros, motivos de status profissional, e motivos de situação de vida, entre os quais se inserem questões de saúde e relativas à maternidade (Konradt et al., 2000).

\section{A Transição e os Desafios do Trabalho a Partir de Casa}

Frente à indagação sobre os aspectos mais desafiadores do trabalho a partir de casa, os participantes sinalizaram os tópicos ligados à família, rotina, relacionamento interpessoal, e relacionamento com o mercado. Entre estes temas, os aspectos relacionados à família foram citados como relevantes para a adaptação e inserção plena no trabalho. Sobre a aceitação de familiares e amigos do trabalho em home-office, 46 participantes relataram que seu grupo de convívio teve aceitação satisfatória, rápida e aco- lhedora. Os participantes relataram, em maioria, que cônjuges, pais e filhos foram figuras de suporte fundamental na adaptação a esse novo modelo de atividade laboral, bem como figuras de respeito às diferenças de horários e à intensidade do trabalho. As respostas dos participantes reforçam a necessidade do comprometimento entre o trabalhador e a família, para que o ambiente de habitação não interfira no laboral e vice-versa, uma vez que a coexistência destes âmbitos tem a possibilidade de promover ou diminuir a satisfação com a escolha de se trabalhar em home-office (Ng, 2010).

Dezessete participantes, contudo, lidaram com a falta de compreensão por parte de seus familiares e amigos, além de 13 participantes que relataram haver sofrido com o não reconhecimento da modalidade de trabalho em home-office. Questões como "reclamações quanto a mudanças na rotina da casa", "dúvidas em relação à capacidade de adaptação do participante", "representação do trabalho como momento de sair de casa" e "trabalho sem as seguranças do regime CLT" se destacaram nas respostas.

Foi citada, também, a representação de que trabalhadores em regime home-office dedicam menos quantidade de horas ao exercício profissional e são menos comprometidos com o trabalho e os resultados que fornecem. Sete home-officers relataram que seus familiares e amigos tiveram dificuldade para compreender os termos da modalidade de trabalho, como expresso por Duarte (2006), em que o contrato de trabalho não difere do realizado para trabalhadores em regime comum, sendo a maior diferença a liberdade que o profissional de home-office possui para administrar seu tempo da maneira que lhe convém.

A dificuldade em estabelecer limites entre o ambiente pessoal e o profissional, entre a casa e o escritório, também foi lembrada como uma desvantagem. Em estudo longitudinal com 267 participantes, Frone, Russel e Cooper (1997) verificaram o impacto da família no trabalho e do trabalho na família, explicitando nos resultados que ambos possuem consequências negativas na saúde dos profissionais. Os resultados revelaram que os conflitos família-trabalho se relacionaram longitudinalmente com o aumento nos níveis de 
depressão e piora na saúde física; em contrapartida, conflitos trabalho-família revelaram, longitudinalmente, aumento no abuso de consumo de bebidas alcoólicas. Troup e Rose (2012) verificaram, em pesquisa com 856 teletrabalhadores australianos, a existência de diferenças entre teletrabalho formal (ligado a uma organização) e informal (autônomo) quanto aos níveis de satisfação em homens e mulheres com filhos. Resultados apontam que o trabalho formal é mais satisfatório e recompensador para a amostra pesquisada, principalmente com relação à satisfação geral com o trabalho e a satisfação com a distribuição das tarefas de cuidado dos filhos.

Com relação ao processo de transição do trabalho em regime regular para o de home-office, 36 participantes relataram que o processo de adaptação foi rápido e tranquilo. Desses, 13 citaram condições que facilitaram a adaptação, entre elas: a adequação do ambiente e dos instrumentos de trabalho, o estabelecimento de rotinas rígidas e o desenvolvimento de habilidades, como disciplina e persistência. Este dado corrobora com o que foi apresentado por Mello (1999), sinalizando que trabalhar em casa não é uma atividade caseira amadora e sim uma atividade de trabalho que exige bom planejamento e dedicação, além de metas claras de desenvolvimento.

Dezoito participantes, contudo, explicitaram terem passado por uma adaptação lenta e difícil ao home-office. Citaram como motivos o sentimento de insegurança inicial, a dificuldade de adaptação da família ao novo modelo de trabalho e a dificuldade com relação às rotinas. Foram citadas, também, a perda de contato com colegas de trabalho e a falta de supervisão, como influências negativas na adaptação ao home-office. Para Fireman (1999), estes fatores influenciam diretamente o interesse em abandonar esse modelo, corroborando a necessidade de flexibilidade para a inserção e adaptação ao home-office.

Em relação às vantagens e desvantagens de se atuar em home-office, os participantes citaram contrapontos entre o lado positivo e o lado negativo de se trabalhar remotamente, conforme apresentado na Tabela 1.

\section{Tabela 1}

Principais Vantagens e Desvantagens de se Trabalhar em Home-Office

\begin{tabular}{llll}
\hline $\begin{array}{l}\text { Principais vantagens de se trabalhar } \\
\text { em home-office }\end{array}$ & & $\begin{array}{l}\text { Principais desvantagens de se trabalhar } \\
\text { em home-office }\end{array}$ \\
\hline Flexibilidade de horários & 49 UR & Falta de socialização & 37 UR \\
Redução/fim do tempo de trânsito & $43 \mathrm{UR}$ & Má divisão do tempo e facilidade de distração & 35 UR \\
Redução de custos & $26 \mathrm{UR}$ & Dificuldade em estabelecer limites entre casa-trabalho & 31 UR \\
Aumento da produtividade & $18 \mathrm{UR}$ & Menor exposição profissional & 19 UR \\
Maior praticidade no dia-a-dia & $17 \mathrm{UR}$ & Custo de manutenção do home-office & 11 UR \\
\hline
\end{tabular}

Nota. UR: Unidades de Registros.

Para Ferreira (2006), a redução dos custos atua em mão dupla, tanto para os trabalhadores quanto para os empregadores, uma vez que é possível diminuir os custos com o deslocamento e com a planta física empresarial. A elevação da produtividade pode ser explicada pela sensação de autoridade e de autonomia que o trabalho em home-office possibilita, ampliando a autoconfiança do trabalhador em suas capacidades. Essa produtividade crescente pode, também, estar conectada à melhor adaptação ao ambiente e aos horários de trabalho, possibilitando ao home-officer que comece sua jornada laboral mais tarde e trabalhe até o período da noite, caso seja esse seu horário de maior disposição (Bailey \& Kurtland, 2002; Konradt et al., 2000).

Sobre as desvantagens de se atuar em home-office, para Rosenfield e Alves (2011) a substituição das relações pessoais e diretas por relações à distância e o isolamento profissional podem acarretar outros prejuízos, como a própria falta de delimitação da definição do trabalho 
em home-office. Em suas respostas, os participantes apontam que o isolamento dos colegas de trabalho interfere na disposição para realizar pausas e para trocar informações profissionais. Ainda que o contato excessivo e as brincadeiras tenham um efeito negativo, a perda da socialização pode promover menor adaptação ao ambiente, além de diminuir as possibilidades de trocas de conhecimentos. A importância das relações não reside somente no contato profissional, pois o vínculo formado pode ser transposto para diferentes espaços e a perda do contato implica perda do sentimento de pertença, tanto do grupo profissional quanto de amizade (Ellison, 1999).

Como contrapontos à vantagem da produtividade foram citadas a má divisão do tempo e a facilidade de distração. Para os participantes, ingressar no ritmo de trabalho do home-office exige elevada disciplina e autonomia, o que acarreta dificuldades iniciais em administrar o tempo e a flexibilidade oferecida. Como explicitado, ainda que o principal atrativo dessa modalidade de trabalho seja a liberdade e a capacidade de se auto-organizar, a falta de tais competências pode impossibilitar a continuidade nesse modelo (Di Martino \& Wirth, 1990; Pyöriä, 2011).

Os participantes vinculados a organizações de trabalho citaram a menor exposição profissional como uma desvantagem do home-office. A perda do contato diário com os superiores no ambiente corporativo e diminuição do feedback espontâneo pode influenciar em processos de aprendizagem, promoções, demissões e culminar em menor percepção de possibilidades de crescimento (Golden et al., 2008). O distanciamento do centro de decisões é visto como um problema inerente ao home-office, que exige do trabalhador o uso de estratégias diversificadas, para se manter presente e atuante, além de facilitar que seja lembrado pela empresa em processos de promoção (Ward \& Shabha, 2001).

Sobre a realização das atividades no ambiente de trabalho em casa, Nix, Ryan, Manly e Deci (1999) sinalizam que quanto maior o nível de autonomia percebido do trabalhador na execução de suas tarefas maiores são os níveis de afetos positivos e de vitalidade percebidos para a execução. No Brasil, Covacs (2006) demonstrou que o valor de autonomia no trabalho impacta positivamente no nível de envolvimento do trabalhador com seu trabalho. Tal aspecto, a partir dos dados levantados no presente estudo, parece demonstrar uma relação positiva entre o home-office e os processos de saúde no trabalho; no entanto, ainda é necessário explorar mais especificamente esse tópico. Segundo Siqueira e Padovan (2008), o bem-estar no trabalho se relaciona à satisfação com as atividades realizadas, com o envolvimento com as tarefas e as demandas, e com o compromisso afetivo com a Organização, sendo necessária a interação entre estes três fatores para promover um nível elevado de bem-estar.

Devido a características de amostragem, tais como a menor representatividade frente à população total e à baixa participação feminina, impactando na distribuição igualitária entre sexos, este estudo se apresenta como uma investigação brasileira inicial sobre a temática do trabalho em home-office, esboçando bases para estudos de continuação e aprofundamento. Sugere-se que novas pesquisas explorem, de modo mais focado, as características necessárias aos trabalhadores para a atuação nessa modalidade, tais como a autonomia e a disciplina para a realização de atividades. Uma vez que os dados coletados para este estudo foram obtidos por coleta online, de respostas abertas, intervenientes, como a baixa disponibilidade para responder a questionários mais longos, podem ter contribuído para o menor número total de participantes.

\section{Considerações Finais}

Aspectos associados à otimização do tempo, obtenção de independência, maior maturidade profissional, diminuição de custos pessoais e organizacionais, além do aumento de produtividade e redução do tempo no trânsito foram aspectos favoráveis e motivadores para o investimento no home-office como modelo de trabalho para os participantes. Entretanto, percebe-se a necessidade de igualar, ou aproximar, o investimento nas esferas pessoal e profissional, facilitando a 
interação entre estas, a fim de diminuir os impactos negativos dos conflitos família-trabalho e trabalho-família.

A adaptação ao ambiente de trabalho, além de uma negociação com a família, em termos dos costumes e do espaço para realização da atividade, reforça o imperativo da formulação de rotinas que equilibrem a realidade do trabalho a partir de casa e partam da realidade do trabalhador. Por estar em processo de inserção e consolidação no cenário brasileiro, o home-office encontra entraves, como a falta de apoio, de reconhecimento e de incentivo à adaptação. De forma semelhante, a cultura vigente no Brasil tende a compreender o trabalho a partir de casa como uma atividade temporária e desqualificada, diminuindo a aceitação por parte dos familiares e dos próprios trabalhadores. Esse fato se mostra um fator que dificulta a consolidação do trabalho em home-office no país. Nesta mesma direção, Bastos e cols. (2013) destacam que os aspectos relativos ao envolvimento e compromisso dos trabalhadores em home-office são pouco estudados. Todavia, apontam que esses aspectos merecem destaque pela sua expansão dentro do modelo de mercado global. Da mesma forma, outras facetas do campo do trabalho, como cultura, desempenho, perfil, suporte, aprendizagem, envolvimento com o trabalho, satisfação, entre outras, merecem investigações mais específicas entre os home-officers.

Mudanças nos aspectos de socialização são um das consequências resultantes do trabalho em home-office: trabalhar de casa promove a diminuição do contato pessoal, direto e diário com pares e superiores e, em contrapartida, aumenta o contato com familiares e cônjuges. Pesquisas futuras podem investigar estratégias de gerenciamento e adaptação desses trabalhadores para administrar a nova dinâmica de relacionamentos, além de buscar informações sobre alterações na qualidade das relações íntimas e profissionais. Nesta direção, políticas de aproximação por parte das empresas podem agir como agregadoras, possibilitando a ampliação da percepção de pertença e da motivação para a atividade laboral. O menor feedback sobre as atividades profissionais pode influenciar na qualidade dos resulta- dos apresentados, sendo interessante que futuras pesquisas busquem conhecer estratégias de autonomia e identifiquem alterações nos níveis dessa competência nos trabalhadores, tendo como base os acompanhamentos de desenvolvimento profissional.

Em contrapartida, os participantes explicitam que o apoio para a inserção no modelo de home-office não é o único ponto a ser considerado para decidir trabalhar nesta modalidade. Características pessoais, como atenção, resistência a frustrações e capacidade para se automotivar também se fazem importantes nos momentos de distância das Organizações e dos colegas de trabalho, podendo ser este um ponto a explorar em futuras pesquisas, dentro da área do trabalho remoto. Sugere-se que futuros trabalhos investiguem a capacidade de atenção e foco nas tarefas, a capacidade de resolução de problemas e o estilo de tomada de decisão dos indivíduos que trabalham remotamente, possibilitando a compreensão de variáveis intervenientes ou preditoras do ajustamento ao home-office.

São inúmeras as modificações geopolíticas e sociais aliadas à crescente escolha mercadológica por meios de prestação de serviços e busca de trabalhadores em outras cidades, estados e países para a realização de atividades específicas. Com isso, faz-se necessário conhecer melhor a realidade do trabalho remoto, delineando uma agenda de pesquisas com os diferentes tópicos na área de Psicologia Organizacional e do Trabalho.

\section{Referências}

Bailey, D. E., \& Kurland, N. B. (2002). A review of telework research: Findings, new directions and lessons for the study of modern work. Journal of Organizational Behavior, 23, 383-400. doi:10.1002/job.144

Bardin, L. (1997). Análise de conteúdo. Lisboa, Portugal: Edições 70.

Baruch, Y. (2001). The status of research on teleworking and agenda for future research. International Journal of Management Reviews, 6(2), 113-129. doi:10.1111/1468-2370.00058 
Bastos, A. V. B., Rodrigues, A. C. A., Moscon, D. C. B., Silva, E. E. C., \& Pinho, A. P. M. (2013). Comprometimento no trabalho: Fundamentos para gestão de pessoas. In L. O. Borges \& L. Mourão (Eds.), O trabalho e a organização: Atuações a partir da Psicologia (pp. 279-310). Porto Alegre, RS: Artmed.

Covacs, J. M. L. M. (2006) Bem-estar no trabalho: O impacto dos valores organizacionais, percepção de suporte organizacional e percepção de justiça (Dissertação de mestrado, Universidade Metodista de São Paulo, São Bernardo do Campo, SP, Brasil).

Di Martino, V., \& Wirth, L. (1990). Telework: A new way of working and living. International Labour Review, 129(5), 529-554.

Duarte, J. B. (2006). O trabalho no domicílio do empregado: Controle da jornada e responsabilidade pelo custeio dos equipamentos envolvidos. Revista ST, 195, Doutrina.

Ellison, N. B. (1999). Social impacts: New perspectives on telework. Social Science Computer Review, 17(3), 338-356.

Ferreira, J. C., Jr. (2006). Teletrabalho: O paradigma de um novo estilo de trabalho. In Anais do XIII Simpósio de Engenharia de Produção. Bauru, SP: Universidade Estadual Paulista "Júlio de Mesquita Filho". Recuperado em http://www. simpep.feb.unesp.br/anais/anais_13/artigos/20. pdf

Fireman, S. M. (1999). A model of telecommuting withdrawal: Employee perceptions predicting the reduction or stopping of telework (Doctoral dissertation, University of Washington).

Frone, M. R., Russell, M., \& Cooper, M. L. (1997). Relation of work-family conflict to health outcomes: A four-year longitudinal study of employed parents. Journal of Occupational and Organizational Psychology, 70(4), 325-335. doi:10.1111/j.2044-8325.1997.tb00652.x

Golden, D., Veiga, J. F., \& Dino, R. N. (2008). The impact of professional isolation on teleworker job performance and turnover intentions: Does time spent teleworking, interacting face-to-face, or having access to communication-enhancing technology matter? Journal of Applied Psychology, 93(6), 1412-1421. doi:10.1037/a0012722

Huws, U., Korte, W. B., \& Robinson, S. (1990). Telework: Towards the elusive office. Chichester, UK: Wiley.
Jaakson, K., \& Kallaste, E. (2010). Beyond flexibility: Reallocation of responsibilities in the case of telework. New Technology, Work and Employment, 25(1), 196-209. doi:10.1111/ j.1468-005X.2010.00248.x

Konradt, U., Schmook, R., \& Mälecke, M. (2000). Impacts of telework on individuals, organizations and families - A critical review. In C. L. Cooper \& I. T. Robertson (Eds.), Organizational psychology and development: A reader for students and practitioner (pp. 339-375). Chichester, UK: Wiley.

Lim, V. K. G., \& Teo, T. S. H. (2000). To work or not to work at home - An empirical investigation of factors affecting attitudes toward teleworking. Journal of Managerial Psychology, 15(6), 560586. doi:10.1108/02683940010373392

Mann, S., \& Holdsworth, L. (2003). The psychological impact of teleworking: Stress, emotions and health. New Technology, Work and Employment, 18(3), 196-211. doi:10.1111/1468-005X.00121

Mello, A. (1999). Teletrabalho (telework): O trabalho em qualquer lugar e em qualquer hora. Rio de janeiro, RJ: Qualitymark.

Mendonça, M. (2010). A inclusão dos "home-officers" no setor residencial no município de São Paulo (Tese de doutorado, Universidade de São Paulo, SP, Brasil).

Ng, C. F. (2010). Teleworker's home office: An extension of corporate office? Facilities, 28(3/4), 137-155. doi:10.1108/02632771011023113

Nix, G. A., Ryan, R. M., Manly, J. B., \& Deci, E. L. (1999). Revitalization through self-regulation: The effects of autonomous and controlled motivation on happiness and vitality. Journal of Experimental Social Psychology, 35(1), 266-284. doi:10.1006/jesp.1999.1382

Pratt, J. H. (1984). Home teleworking: A study of its pioneers. Technological Forecasting and Social Change, 25(1), 1-14.

Pyöriä, P. (2011). Managing telework: Risks, fears and rules. Management Research Review, 34(4), 386-399. doi:10.1108/01409171111117843

Rasmussen, E., \& Corbett, G. (2008). 'Why isn't teleworking working?' New Zealand Journal of Employment Relations, 33(2), 20-32.

Rosa, M. D. (2010). A globalização e a organização do processo de trabalho. Cadernos da Escola de Direito e Relações Internacionais, 13(1), 496-513. 
Rosenfield, C. L., \& Alves, D. A. (2011). Autonomia e trabalho informacional: O teletrabalho. Revista de Ciências Sociais, 54(1), 207-233. doi:10.1590/S0011-52582011000100006

Sardeshmukh, S. R., Sharma, D., \& Golden, T. D. (2012). Impact of telework on exhaustion and job engagement: A job demands and job resources model. New Technology, Work and Employment, 27(3), 193-207. doi:10.1111/ j.1468-005X.2012.00284.X

Siqueira, M. M. M., \& Padovam, V. A. R. (2008). Bases teóricas de bem-estar subjetivo, bem-estar psicológico e bem-estar no trabalho. Psicologia: Teoria e Pesquisa, 24(2), 201-209.

Strauss, A., \& Corbin, J. (1998). Basics of qualitative research: Techniques and procedures for developing grounded theory ( $2^{\text {nd }}$ ed.). London: Sage.

Tremblay, D. G., Paquet, R., \& Najem, E. (2006). Telework: A way to balance work and family or to increase work-family conflict? Canadian Journal of Communication, 31(3), 715-731.
Troup, C., \& Rose, J. (2012). Working from home: Do formal or informal telework arrangements provide better work-family outcomes? Community, Work \& Family, 15 (4), 471-486. doi:10.10 $80 / 13668803.2012 .724220$

Wachelke, J. F. R., \& de Andrade, A. L. (2009). Influência do recrutamento de participantes em sítios temáticos e comunidades virtuais nos resultados de medidas psicológicas aplicadas pela Internet. Psicologia: Teoria e Pesquisa, 25(3), 357-367. doi:10.1590/S0102-37722009000300009

Ward, N., \& Shabba, G. (2001). Teleworking: An assessement of socio-psychological factors. Facilities, 19(1/2), 61-70. 